\title{
Corrigendum to "Suppression of Dynamical Network Biomarker Signals at the Predisease State (Mibyou) before Metabolic Syndrome in Mice by a Traditional Japanese Medicine (Kampo Formula) Bofutsushosan"
}

\author{
Keiichi Koizumi $\mathbb{D}^{1,2}$ Makito Oku $\mathbb{D}^{3,4}$ Shusaku Hayashi ${ }^{3},{ }^{5,6}$ Akiko Inujima $\mathbb{D}^{1,2}$ \\ Naotoshi Shibahara $\mathbb{D}^{1},{ }^{1}$ Luonan Chen $\mathbb{D}^{7,8}$ Yoshiko Igarashi ${ }^{7},{ }^{9}$ Kazuyuki Tobe $\mathbb{D}^{9}$, \\ Shigeru Saito $\mathbb{D}^{10},^{10}$ Makoto Kadowaki $\mathbb{D},{ }^{5}$ and Kazuyuki Aihara $\mathbb{D}^{8,11}$ \\ ${ }^{1}$ Division of Kampo Diagnostics, Institute of Natural Medicine, University of Toyama, Toyama, Japan \\ ${ }^{2}$ Laboratory of Drug Discovery and Development for Pre-disease, Section of Host Defences, Division of Bioscience, \\ Institute of Natural Medicine, University of Toyama, Toyama, Japan \\ ${ }^{3}$ Division of Chemo-Bioinformatics, Institute of Natural Medicine, University of Toyama, Toyama, Japan \\ ${ }^{4}$ Laboratory of Chemo-Bioinformatics, Section of Host Defences, Division of Bioscience, Institute of Natural Medicine, \\ University of Toyama, Toyama, Japan \\ ${ }^{5}$ Division of Gastrointestinal Pathophysiology, Institute of Natural Medicine, University of Toyama, Toyama, Japan \\ ${ }^{6}$ Laboratory of Gastrointestinal Disorder, Section of Host Defences, Division of Bioscience, Institute of Natural Medicine, \\ University of Toyama, Toyama, Japan \\ ${ }^{7}$ CAS Center for Excellence in Molecular Cell Science, Institute of Biochemistry and Cell Biology, \\ Shanghai Institutes for Biological Sciences, Chinese Academy of Sciences, Shanghai, China \\ ${ }^{8}$ Institute of Industrial Science, The University of Tokyo, Tokyo, Japan \\ ${ }^{9}$ First Department of Internal Medicine, Faculty of Medicine, University of Toyama, Toyama, Japan \\ ${ }^{10}$ University of Toyama, Toyama, Japan \\ ${ }^{11}$ International Research Center for Neurointelligence (WPI-IRCN), The University of Tokyo Institutes for Preemptive Study, \\ The University of Tokyo, Tokyo, Japan
}

Correspondence should be addressed to Keiichi Koizumi; kkoizumi@inm.u-toyama.ac.jp

Received 17 April 2021; Accepted 17 April 2021; Published 24 April 2021

Copyright (C) 2021 Keiichi Koizumi et al. This is an open access article distributed under the Creative Commons Attribution License, which permits unrestricted use, distribution, and reproduction in any medium, provided the original work is properly cited.

In the article titled "Suppression of Dynamical Network Biomarker Signals at the Predisease State (Mibyou) before Metabolic Syndrome in Mice by a Traditional Japanese Medicine (Kampo Formula) Bofutsushosan" [1], the authors wish to clarify that the DNB scores of the untreated group shown in blue in Figure 3 were recalculated from data obtained from the Gene Expression Omnibus (GEO) repository, accession number: GSE112653 [2], and were presented for comparison. The authors confirm that this does not affect the results and conclusions of the article.

\section{References}

[1] K. Koizumi, M. Oku, S. Hayashi et al., "Suppression of dynamical network biomarker signals at the predisease state (Mibyou) before metabolic syndrome in mice by a traditional Japanese medicine (Kampo formula) bofutsushosan," Evidence-Based Complementary and Alternative Medicine, vol. 2020, p. 9129134, 2020.

[2] K. Koizumi, M. Oku, S. Hayashi et al., "Identifying pre-disease signals before metabolic syndrome in mice by dynamical network biomarkers," Scientific Reports, vol. 9, p. 8767, 2019. 